\title{
The Effect of Birthweight on Tooth-Size Variability in Twins
}

\author{
Mary V. B. Apps, Toby E. Hughes, and Grant C. Townsend \\ Dental School,The University of Adelaide, Australia
}

S tudies indicate that low birthweight (LBW) children display reduced deciduous tooth size but there is little information about permanent tooth size. It has also been shown that dental fluctuating asymmetry (FA) increases in response to various environmental influences, but the relationship between birthweight and FA remains unclear. The aim of this study was to compare tooth size and asymmetry, according to birthweight, in the deciduous and permanent dentitions of a sample of Australian twins. The study sample comprised 436 twins, classified into 2 groups: normal birthweight (NBW > 2500g) and low birthweight (LBW $\leq 2500 \mathrm{~g})$. For each individual it was generally possible to measure maximum mesiodistal crown diameters of both deciduous and permanent central incisors from serial dental models. Correlations were calculated between tooth-size variables and birthweight; subsequently comparisons of tooth size and FA were made between the LBW and NBW samples using Student's $t$ tests. Small positive correlations (around .1) were noted between birthweight and tooth-size variables. There was no evidence of tooth-size reduction in the LBW male sample, but the LBW females displayed tooth-size reduction of approximately 2-3\% for both deciduous and permanent incisors, compared to the NBW females. There was no evidence of increased FA in the LBW individuals of either sex. These findings indicate that developing teeth are generally well-protected from developmental disturbances during prenatal and perinatal periods. Further research is needed to clarify the biological basis of an apparently true but weak association between tooth size and birthweight.

Tooth morphology and size conform to a polygenic mode of inheritance with both genetic factors and environmental influences contributing to observed variability (Dempsey \& Townsend, 2001). While genetic influences seem to predominate, factors such as maternal health status and maternal smoking during pregnancy can affect human odontogenesis (Garn et al., 1979; Heikkinen et al., 1995). Premature birth and low birthweight have also been suggested to affect dental development and low birthweight (LBW) children have been shown to have reduced tooth size in the deciduous dentition (Garn et al., 1979; Fearne \& Brook, 1993; Seow \& Wan, 2000). As the crown morphology of deciduous teeth is determined predominantly in the prenatal period, it might be expected that these teeth would be more affected by prenatal environmental factors than the permanent teeth, which calcify postnatally. However, Garn et al. (1979) reported that prenatal disturbances can lead to alteration of permanent tooth morphology to a degree comparable with that of the deciduous teeth. There is still little information available on the nature and extent of associations between birthweight and tooth size, particularly in the permanent dentition.

It is rare for antimeric teeth to be perfectly symmetrical, despite the assumption that the genetic information is identical for teeth on either side of the midline. It is a corollary of this assumption that the influence of 'environmental' factors (which by default includes everything other than the Mendelian factors included in the model at hand) on tooth development explains why antimeres do not develop as exact mirror images (Heikkinen et al., 1998). Odontometric studies commonly refer to two types of asymmetry: directional asymmetry (DA) and fluctuating asymmetry (FA). DA describes an asymmetry where average left-right difference in dental-crown size is significantly different from zero, and FA refers to the random variations around that mean directional value that occur between corresponding teeth on opposite sides of the dental arch (Townsend et al., 1999). The degree of FA is often used as a measure of developmental stability, increased FA being thought to reflect greater developmental disturbances or a reduced capacity to buffer environmental perturbations (Van Valen, 1962). Elevated FA has been observed in individuals with congenital abnormalities and genetic syndromes (Townsend, 1983), as well as numerous environmental disturbances such as smoking and

Received 3 May, 2004; accepted 20 July, 2004.

Address for correspondence: Professor G. C. Townsend, Professor of Dental Science, Dental School, The University of Adelaide, Adelaide, South Australia 5005, Australia. E-mail: grant.townsend@adelaide. edu.au 
alcohol consumption during pregnancy (Kieser, 1992; Kieser et al., 1997).

Boklage (1984), using permanent dental-crown diameters as a model system for craniofacial development, found that monozygotic (MZ), dizygotic (DZ) and singleton groups differed significantly in tooth-size distributions, suggesting that they were developmentally distinct groups. Boklage went on to note that twins of both zygosities were more symmetrical for tooth size than singletons, the opposite relationship to what one might expect if there were a simple, direct relationship between developmental stress and dental asymmetry. In fact, the central incisors showed the greatest twin-singleton differences in dental fluctuating asymmetry (Boklage, 1987).

The main aim of this study was, therefore, to compare deciduous and permanent tooth size and dental asymmetry, according to birthweight, in a sample of twins. One hypothesis was that the LBW twins would have smaller deciduous teeth, but that their permanent teeth would not be affected to the same extent, if at all. Another hypothesis was that the LBW twins would have higher levels of FA than twins of normal birthweight.

\section{Materials and Methods}

The study sample comprised 436 twins who were participants in an ongoing study of dental and facial development in Australian twins and their families. None of the participants included in this study had serious medical problems. The sample consisted of 220 females and 216 males. Of the participants, 184 were $\mathrm{MZ}$ twins and 252 were DZ twins. Twin zygosities were confirmed by the analysis of DNA obtained from buccal cells (Hughes et al., 2001). Although comparisons of $\mathrm{MZ}$ twin pairs and $\mathrm{DZ}$ twin pairs provide an opportunity to quantify the relative contributions of genetic and environmental influences to observed variation, the focus of this study was on the relationship of tooth size to birthweight, not on the genetic basis to variation which has been the subject of other recent publications (Dempsey \& Townsend, 2001; Hughes et al., 2001). Given that twins tend to be premature or display LBW more often than singletons (Liu \& Blair, 2002), the twin sample was particularly appropriate for examining the association between birthweight and tooth size. However, given Boklage's $(1984,1987)$ earlier findings of differences in dental distributions between twins and singletons, any findings in twins may not be applicable to singletons.

A birthweight classification system, similar to that employed by Seow et al. (1988), was used in this study. The twins were classified into two groups: normal birthweight (NBW $>2500 \mathrm{~g}$ ) and low birthweight $(\mathrm{LBW} \leq 2500 \mathrm{~g})$. Of the total sample, 199 individuals were classified as LBW, while the remaining 237 were considered to be NBW. Table 1 shows the distribution of subjects between the birthweight

\section{Table 1}

Distribution of Low Birthweight (LBW $\leq 2500 \mathrm{~g}$ ) and Normal Birthweight (NBW $>2500 \mathrm{~g}$ ) in the Different Zygosity Groups

\begin{tabular}{lrrr}
\hline & LBW & NBW & Total \\
\hline MZ male & $39(41 \%)$ & $57(59 \%)$ & 96 \\
MZ female & $51(58 \%)$ & $37(42 \%)$ & 88 \\
DZ male & $35(46 \%)$ & $41(54 \%)$ & 76 \\
DZ female & $41(47 \%)$ & $47(53 \%)$ & 88 \\
DZ opp. sex male & $15(34 \%)$ & $29(66 \%)$ & 44 \\
DZ opp. sex female & $18(41 \%)$ & $26(59 \%)$ & 44 \\
Total & $199(46 \%)$ & $237(54 \%)$ & 436 \\
\hline
\end{tabular}

categories and the different twin groups: monozygotic and dizygotic (same-sexed and opposite-sexed).

Dental models obtained at two different stages of development were available for each child. The first set of models was made from impressions obtained at around the age of 6 years, so these models generally showed the deciduous dentition as well as permanent first molars. The second models were made from impressions obtained around the age of 8 to 9 years, so these showed the early mixed dentition, with at least the permanent maxillary and mandibular central incisors present. Accordingly, for each individual it was generally possible to measure both deciduous and permanent central incisors on left and right sides.

Maximum mesiodistal (MD) crown diameters were measured for both the deciduous and permanent central incisors following the method described by Townsend (1983). Only those teeth that were fully erupted, not noticeably affected by tooth wear, and without caries or restorations were selected for measurement. Although central incisors were fully erupted in the 8- to 9-year-old twins, lateral incisors were not included because most permanent maxillary lateral incisors were either unerupted or only partially erupted in the second set of study models. Modified digital callipers were used to measure the MD dimensions to an accuracy of $0.1 \mathrm{~mm}$. Double determinations were performed on the dental models of 30 randomly selected subjects to quantify measurement errors. Student's paired $t$ test was used to assess the significance of the means of differences between first and second measurements and there was no evidence of systematic error $(p>.05)$. Measurement errors, as indicated by Dahlberg's statistic, ranged in value from $0.04 \mathrm{~mm}$ to $0.08 \mathrm{~mm}$ for the mesiodistal dimensions (Dahlberg, 1940). Experimental errors were therefore small, and unlikely to bias the results of the study.

Paired $t$ tests provided a sensitive and appropriate means of assessing whether significant DA was present in the sample (Boklage, 1992), whereas FA was quantified by calculating the absolute value of $(\mathrm{R}-\mathrm{L}) /[(\mathrm{R}+\mathrm{L}) / 2]$, where $\mathrm{R}$ and $\mathrm{L}$ represent the mesiodistal crown diameters of corresponding right and left central incisors (Townsend et al., 1999; 
Corruccini et al., in press), and also by calculating the variance of $(\mathrm{R}-\mathrm{L}) /(\mathrm{R}+\mathrm{L})$ following Sofaer (1979).

Student's $t$ tests were used to compare mean values for tooth size and FA between NBW and LBW samples, while $F$-tests were used to compare variances. Pearson correlation coefficients were calculated between tooth size and birthweight. The significance level was set as $p<.05$.

\section{$\overline{\text { Results }}$}

Estimates of skewness and kurtosis failed to indicate any major deviations from normality in the distributions of tooth-size variables. Accordingly, descriptive statistics including mean values and standard deviations were used in the analysis of tooth size according to birthweight. Initially comparisons between LBW and NBW individuals were made within each of the different twin groups: monozygous females and males, dizygous same-sexed females and males, and dizygous opposite-sexed females and males. As no distinct trends were noted between the twin groups, tooth-size data for the twin groups were combined to maximise sample sizes (Table 2). There was no evidence of a reduction in tooth crown size related to birthweight in the males. However, the mean crown size for LBW females was significantly smaller $(p<$ $.05)$ than that of the NBW females for all of the teeth measured. This suggests that females were more susceptible than males to a reduction in tooth size associated with low birthweight. Mean tooth-size measurements for LBW females were all significantly

Table 2

Descriptive Statistics for Tooth Size in NBW and LBW Groups

\begin{tabular}{llcccccc}
\hline & & \multicolumn{3}{c}{ NBW } & \multicolumn{3}{c}{ LBW } \\
Group & Tooth & $N$ & $\bar{x}$ & $S D$ & $N$ & $\bar{x}$ & $S D$ \\
\hline Male & r max I1 & 41 & 8.52 & 0.49 & 38 & 8.54 & 0.49 \\
& I max I1 & 40 & 8.54 & 0.51 & 37 & 8.51 & 0.52 \\
& r man I1 & 41 & 5.27 & 0.35 & 36 & 5.31 & 0.31 \\
& I man I1 & 41 & 5.33 & 0.35 & 36 & 5.31 & 0.34 \\
& r maxi1 & 76 & 6.34 & 0.39 & 68 & 6.32 & 0.32 \\
& I max i1 & 75 & 6.32 & 0.37 & 65 & 6.26 & 0.35 \\
& r man i1 & 53 & 3.97 & 0.25 & 52 & 3.95 & 0.25 \\
& I man i1 & 58 & 3.95 & 0.27 & 52 & 3.97 & 0.24 \\
Female & r max I1 & 46 & 8.54 & 0.53 & 42 & $8.28^{1}$ & 0.51 \\
& I max I1 & 46 & 8.53 & 0.50 & 41 & $8.26^{1}$ & 0.47 \\
& r man I1 & 46 & 5.29 & 0.30 & 40 & $5.16^{1}$ & 0.29 \\
& I man I1 & 45 & 5.30 & 0.33 & 40 & $5.14^{1}$ & 0.30 \\
& r maxi1 & 85 & 6.33 & 0.40 & 78 & $6.18^{1}$ & 0.35 \\
& I max i1 & 85 & 6.25 & 0.38 & 78 & $6.10^{1}$ & 0.33 \\
& r man i1 & 60 & 3.92 & 0.27 & 58 & $3.82^{1}$ & 0.26 \\
& I man i1 & 62 & 3.92 & 0.30 & 57 & $3.82^{1}$ & 0.24 \\
\hline
\end{tabular}

Note: 'indicates that the mean value of LBW group is significantly smaller than the mean value of the NBW control group at $p<.05$.

${ }^{2} \mid 1$ represents permanent central incisor

i1 represents deciduous central incisor

$\max =$ maxillary

man $=$ mandibular
Table 3

FA Scores in LBW and NBW Groups

(a)

\begin{tabular}{lcccccc} 
& \multicolumn{3}{c}{ NBW } & \multicolumn{3}{c}{ LBW } \\
Tooth & $N$ & $\overline{\mathrm{x}}$ & $S D$ & $N$ & $\overline{\mathrm{x}}$ & $S D$ \\
\hline max I1 & 84 & 1.33 & 1.30 & 77 & 1.47 & 1.36 \\
man I1 & 85 & 2.15 & 1.83 & 73 & 1.94 & 1.67 \\
max i1 & 153 & 1.99 & 1.67 & 136 & 2.00 & 1.63 \\
man i1 & 110 & 2.33 & 2.30 & 105 & $2.75^{1}$ & 2.38 \\
\hline
\end{tabular}

Note: $\bar{x}=100 x$ mean of the absolute value of $(R-L) /[(R+L) / 2]$

1 Value significantly greater in LBW group compared with NBW group at $p<.05$.

\begin{tabular}{lrrrr}
\hline (b) & \multicolumn{2}{c}{ NBW } & \multicolumn{2}{c}{ LBW } \\
Tooth & $N$ & $\mathrm{~V}\left(\times 10^{4}\right)$ & $N$ & $\mathrm{~V}\left(\times 10^{4}\right)$ \\
\hline max I1 & 77 & 1.02 & 84 & 0.88 \\
man I1 & 73 & 1.66 & 85 & 1.93 \\
max i1 & 136 & 1.44 & 153 & 1.49 \\
man i1 & 105 & 3.32 & 110 & 2.70 \\
\hline
\end{tabular}

Note: $V=$ Variance $(\mathrm{R}-\mathrm{L}) /(\mathrm{R}+\mathrm{L})$

smaller $(p<.05)$ than those for LBW males. There were no significant differences between the mean values for tooth size of NBW females compared with NBW males.

Initial testing showed no evidence of any significant directional dental asymmetry. Furthermore, no systematic trends were evident in the FA between sexes or zygosity groups, so FA values are presented for the males and females and the different twin groups combined. Table $3 \mathrm{a}$ presents mean FA scores derived from the absolute values of $(\mathrm{R}-\mathrm{L}) /[(\mathrm{R}+\mathrm{L}) / 2]$ for the deciduous and permanent central incisors in the LBW and NBW groups. Although FA scores were all, by definition, greater than zero, the distributions conformed generally to the upper half of normal distributions. Greater FA was noted in three of the four comparisons, but statistical significance was only shown in one of these comparisons (for the deciduous mandibular central incisor). Table $3 \mathrm{~b}$ shows the variance estimates of FA, none of which differed significantly between the LBW and NBW groups. There was therefore no clear evidence that low birthweight was associated with increased FA in our sample.

Pearson correlation coefficients between birthweight and tooth size in the females were all greater than zero for both deciduous and permanent central incisors, indicating a small positive association between these variables (Table 4). However, none of the correlations were statistically significant. For the males, four of the eight correlations were negative and only one of the three positive correlations was statistically significant $(p<.05)$. These findings should be viewed with caution, taking into consideration the general lack of statistical significance, but there is some suggestion that tooth size and birth- 
Table 4

Values of Correlation Coefficients between Birthweight (BW) and Tooth-Size Variables

\begin{tabular}{|c|c|c|c|c|c|c|c|c|c|}
\hline & BW & $\mathrm{r} \max \mid 1$ & $|\max | 1$ & $\mathrm{r} \operatorname{man} \mathrm{I1}$ & I man I1 & $r \max i 1$ & I max i1 & r man i1 & I man i1 \\
\hline BW & 1.00 & -.08 & -.06 & -.12 & -.01 & $.19^{1}$ & .15 & .07 & .00 \\
\hline$r \max I 1$ & .18 & 1.00 & $.95^{2}$ & $.67^{2}$ & $.67^{2}$ & $.42^{2}$ & $.38^{2}$ & $.33^{2}$ & .291 \\
\hline$|\max | 1$ & .20 & $.95^{2}$ & 1.00 & $.74^{2}$ & $.75^{2}$ & $.48^{2}$ & $.44^{2}$ & $.40^{2}$ & $.34^{2}$ \\
\hline r man I1 & .18 & $.73^{2}$ & $.74^{2}$ & 1.00 & $.90^{2}$ & .21 & .21 & $.25^{1}$ & .18 \\
\hline I man I1 & .18 & $.78^{2}$ & $.80^{2}$ & $.91^{2}$ & 1.00 & $.28^{1}$ & $.29^{1}$ & $.32^{2}$ & $.26^{1}$ \\
\hline r max i1 & .09 & $.66^{2}$ & $.59^{2}$ & $.58^{2}$ & $.63^{2}$ & 1.00 & $.90^{2}$ & $.72^{2}$ & $.70^{2}$ \\
\hline I max i1 & .13 & $.69^{2}$ & $.65^{2}$ & $.61^{2}$ & $.66^{2}$ & $.93^{2}$ & 1.00 & $.74^{2}$ & $.73^{2}$ \\
\hline $\mathrm{r}$ man i1 & .07 & $.66^{2}$ & $.64^{2}$ & $.63^{2}$ & $.62^{2}$ & $.75^{2}$ & $.74^{2}$ & 1.00 & $.89^{2}$ \\
\hline I man i1 & .08 & $.65^{2}$ & $.59^{2}$ & $.64^{2}$ & $.64^{2}$ & $.78^{2}$ & $.75^{2}$ & $.86^{2}$ & 1.00 \\
\hline
\end{tabular}

Note: Values for males in upper half of matrix and values for females in lower half of matrix.

1 Significant at $p<.05$

${ }^{2}$ Significant at $p<.01$

weight were not as closely correlated within males in our sample as they were within females. Pearson correlation coefficients between right and left antimeres were consistently positive, with the majority of coefficients being significant at $p<.01$. The correlation coefficients between deciduous teeth and their permanent successors, as well as those between corresponding maxillary and mandibular teeth, were also positive and significant at $p<.01$.

\section{Discussion}

While there was no evidence of tooth-size reduction related to birthweight in the males of our sample, tooth size reduction in the LBW females was consistent and was statistically significant for seven of the eight tooth measurements, affecting both the deciduous and permanent central incisors. This finding suggests that females may be more sensitive to reductions in tooth size related to birthweight than males. Such a difference between males and females has not been reported previously. The Pearson correlation coefficients also provide some suggestion that the association between birthweight and tooth size was weaker in the males of our sample than in the females. This finding is interesting because it is not consistent with the theory that the presence of two $\mathrm{X}$ chromosomes in females buffers females better than males against environmental disturbances (Townsend \& Brown, 1980).

Fluctuating asymmetry is considered to be a good measure of developmental stability, greater values of FA being associated generally with greater developmental instability. However, Boklage's (1987) finding that twinning, involving both $\mathrm{MZ}$ and $\mathrm{DZ}$ twins, was associated with a reduction in dental FA compared with singletons indicated that the relationship between 'stress' and FA might not be straightforward in twins. It is, therefore, interesting to note that there were no noticeable differences between the FA levels of the NBW and LBW females in this study, given that the tooth-size reduction observed in the LBW females is thought to be a reflection of disturbance to the environmental homeostasis of tooth formation. It may also be more than coincidental that the permanent tooth class measured in this study, the central incisors, showed the most marked reduction in FA in twins compared with singletons in Boklage's (1987) study.

The reduction in tooth size in LBW females ranged from approximately $2-3 \%$, a smaller reduction than those reported in previous studies. For example, Fearne and Brook (1993) reported size reductions in the order of $4 \%$, and Seow and Wan (2000) reported $6-11 \%$ reductions in the size of deciduous tooth crowns of LBW individuals. One reason for this finding could be the increased developmental stability of the central incisors compared with other teeth such as maxillary lateral incisors which displayed $11 \%$ size reductions in the study by Seow and Wan (2000). Increased developmental stability of the central incisors is in accordance with Butler's morphogenetic field concept (Butler, 1939), whereby stable key teeth are considered to be present within the four morphogenetic fields of the dentition: incisor, canine, premolar and molar. The key tooth is usually the most mesial in each field, with the teeth further distal in a field being increasingly variable in size and morphology, although the mandibular incisor field differs as the lateral incisor is considered the key tooth (Dahlberg, 1945). Only central incisors were measured in this study. Correlation analysis suggested a stronger association between birthweight and deciduous tooth size compared to that between birthweight and permanent tooth size.

The smaller degree of size reduction displayed by our sample of Australian twins compared to previous studies could also relate to the fact that very few of the twins in the current study could be considered to have a very low birthweight (VLBW). Only six of the children in our study weighed less than $1500 \mathrm{~g}$ at birth and would therefore be classed as VLBW according to the classification system used by Seow et al. (1988). It 
is because of this demographic feature that only two categories of birthweight were used in this study: LBW and NBW. Approximately $30 \%$ of the children in the study by Seow and Wan (2000) were categorised as VLBW compared to $1.4 \%$ of the current study participants. A dose-response pattern has been described for tooth-size reduction related to birthweight, with VLBW children showing a greater reduction in deciduous tooth than LBW children (Seow \& Wan, 2000).

The stage of tooth development at the time of an environmental disturbance will determine whether the size and shape of a particular tooth crown will be affected. The tooth germs of the deciduous central incisors are fully formed at around 3-4 months of foetal life, with dentinogenesis beginning at around the fourth to sixth month of foetal life. Crown formation is completed postnatally at around 2-3 months of age (Lunt \& Law, 1974). The tooth germs of the permanent central incisors are fully formed much later, at about 30 weeks of foetal life, with dentinogenesis beginning around 3-4 months of age. Crown formation of these teeth is not completed until about 4-5 years of age (Hillson, 1996).

Various mechanisms could explain the association of reduction in tooth size with low birthweight. The majority of LBW children are born prematurely, that is, prior to reaching 37 weeks of gestation (Fearne \& Brook, 1993). In such a situation the reduced prenatal period of development could itself constitute an environmental disturbance to tooth development. If the gestational period were decreased to the extent that the organs were immature at the time of birth, the disturbances during the perinatal period might disrupt environmental homeostasis during the period of tooth formation.

While genetic factors contribute considerably to tooth-size variation, environmental effects can also have an impact and this was confirmed in our study, although the reduction in tooth size in LBW females was only of the order $2-3 \%$. Our hypothesis, however, that the LBW individuals would have smaller deciduous teeth but that the permanent teeth would be unaffected or affected to a lesser extent, was not fully supported. Instead, size reductions of similar magnitude were observed in the deciduous and permanent incisors of the LBW twins, and only in females. Although the apparent differences in tooth size and birthweight association between the males and females in this study could be due to a sampling effect, our finding is novel and warrants further investigation. The study results did not support the second hypothesis either, as no systematic differences were evident in the fluctuating dental asymmetry values of the LBW group compared with the NBW group. Our findings are, however, not entirely unexpected given that the central incisors of twins appear to be highly symmetrical (Boklage, 1987).
This study indicates that the developing teeth are generally well-protected from environmental disturbances during the prenatal and perinatal periods. More investigations are needed to clarify the nature of the underlying biological basis of an apparently true but weak association between tooth size and birthweight in humans, and to further explore the nature and extent of associations between dental asymmetry and the twinning process.

\section{Acknowledgments}

This study was supported by a grant awarded to Mary Apps by the Australian Dental Research Foundation Inc. and by a grant from the National Health and Medical Research Council of Australia (Competing Epidemiological Grant 157904). Thanks to Mrs Wendy Schwerdt for her assistance with data analysis.

\section{References}

Boklage, C. (1984). Differences in protocols of craniofacial development related to twinship and zygosity. Journal of Craniofacial Genetics and Developmental Biology, 4, 151-169.

Boklage, C. (1987). Developmental differences between singletons and twins in distributions of dental diameter asymmetries. American Journal of Physical Anthropology, 74, 319-331.

Boklage, C. (1992). Method and meaning in the analysis of developmental asymmetries. Journal of Human Ecology (Special Issue), 2, 147-156.

Butler, P. (1939). Studies of the mammalian dentition: Differentiation of the post-canine dentition. Proceedings of the Zoological Society of London, 109B, 1-36.

Corruccini, R., Townsend, G., \& Schwerdt W. (in press). Correspondence between enamel hypoplasia and odontometric bilateral asymmetry in Australian twins. American Journal of Physical Anthropology.

Dahlberg, G. (1940). Statistical Methods for Medical and Biological Students (pp. 122-132). London: Allen and Unwin.

Dahlberg, A. (1945) The changing dentition of man. Journal of the American Dental Association, 32, 676-90.

Dempsey, P., \& Townsend, G. (2001). Genetic and environmental contributions to variation in human tooth size. Heredity, 86, 685-693.

Fearne, J., \& Brook, A. (1993). Small primary toothcrown size in low birthweight children. Early Human Development, 33, 81-90.

Garn, S., Osborne, R., \& McCabe, K. (1979). The effect of prenatal factors on crown dimensions. American Journal of Physical Anthropology, 51, 665-678.

Heikkinen, T., Alvesalo, L., \& Osborne, R. (1995). Maternal smoking during pregnancy reduces selectively intercuspal distances of the first permanent molar. In R. Radlanski \& H. Renz (Eds.), Proceedings 
of the 10th International Symposium on Dental Morphology (pp. 116-121). Berlin, Germany: C \& M Brunne.

Heikkinen, T., Alvesalo, L., Osborne, R., Tienari, J., \& Virtanen, J. (1998). The effect of sex and race on the symmetry of the permanent tooth clinical eruption. In J. Mayhall \& T. Heikkinen (Eds.), Dental Morphology 1998 (pp. 473-482). Oulu, Finland: Oulu University Press.

Hillson, S. (1996). Dental Anthropology. Cambridge: Cambridge University Press.

Hughes, T., Thomas, C., Richards, L., \& Townsend, G. (2001). A study of occlusal variation in the primary dentition of Australian twins and singletons. Archives of Oral Biology, 46, 857-864.

Kieser, J. (1992). Fluctuating odontometric asymmetry and maternal alcohol consumption. Annals of Human Biology, 19, 513-520.

Kieser, J., Groeneveld, H., \& Da Silva, P. (1997). Dental asymmetry, maternal obesity and smoking. American Journal of Physical Anthropology, 102, 133-139.

Liu, Y. C., \& Blair, E. M. (2002). Predicted birthweight for singletons and twins. Twin Research, 5, 529-537.
Lunt, R., \& Law, D. (1974). A review of the chronology of calcification of deciduous teeth. Journal of the American Dental Association, 89, 599-606.

Seow, W., Humphrys, C., Mahanonda, R., \& Tudehope, D. (1988). Dental eruption in low birth-weight prematurely born children: A controlled study. Pediatric Dentistry, 10, 39-42.

Seow, W., \& Wan, A. (2000). A controlled study of the morphometric changes in the primary dentition of pre-term, very-low-birthweight children. Journal of Dental Research, 79, 63-69.

Sofaer, J. (1979). Human tooth-size asymmetry in cleft lip with or without cleft palate. Archives of Oral Biology, 24, 141-146.

Townsend, G. (1983). Fluctuating dental asymmetry in Down's syndrome. Australian Dental Journal, 28, 39-44.

Townsend, G., \& Brown, T. (1980). Dental asymmetry in Australian Aboriginals. Human Biology, 52, 661-673.

Townsend, G., Dempsey, P., \& Richards, L. (1999). Asymmetry in the deciduous dentition: Fluctuating and directional components. Perspectives in Human Biology, 4, 45-52.

Van Valen, L. (1962). A study of fluctuating asymmetry. Evolution, 16, 125-142. 